\title{
BMJ Open Effects of maternal B12 supplementation on neurophysiological outcomes in children: a study protocol for an extended follow-up from a placebo randomised control trial in Bangalore, India
}

\author{
Shilpa Anand, ${ }^{1}$ Susan Thomas, ${ }^{1}$ Mahesh Jayachandra, ${ }^{1}$ Tinku Thomas, ${ }^{2}$ \\ Tor Arne Strand, ${ }^{3}$ Anura V Kurpad, ${ }^{4}$ Christopher P Duggan, ${ }^{5}$ \\ Krishnamachari Srinivasan ${ }^{6}$
}

To cite: Anand S, Thomas S, Jayachandra $\mathrm{M}$, et al. Effects of maternal B12 supplementation on neurophysiological outcomes in children: a study protocol for an extended follow-up from a placebo randomised control trial in Bangalore, India. BMJ Open 2019;9:e024426. doi:10.1136/ bmjopen-2018-024426

- Prepublication history for this paper is available online. To view these files, please visit the journal online (http://dx.doi. org/10.1136/bmjopen-2018024426).

Received 25 May 2018

Revised 25 September 2018

Accepted 6 December 2018

Check for updates

(C) Author(s) (or their employer(s)) 2019. Re-use permitted under CC BY-NC. No commercial re-use. See rights and permissions. Published by BMJ.

For numbered affiliations see end of article.

Correspondence to

Dr Krishnamachari Srinivasan; srinivas@sjri.res.in

\section{ABSTRACT}

Introduction Vitamin B12 deficiency is highly prevalent in pregnant Indian women. Neuropsychological tests have shown an association between low maternal vitamin B12 status and poorer cognitive performances in the offspring, although findings from these studies have been inconsistent. Vitamin B12 has an important role in the formation of myelin which is important for the transmission speed of neural impulses and myelination in the central nervous system has been linked to cognition. Assessing neurophysiological measures using event-related potentials (ERPs) in children may provide additional information on the effect of maternal vitamin B12 supplementation on offspring brain function. The study examines the effects of oral vitamin B12 daily supplements $(50 \mu \mathrm{g})$ to pregnant Indian women on child neurophysiological function at 72 months.

Methods and analysis We previously conducted a double-blind, placebo-controlled study to examine the effects of maternal vitamin B12 supplementation on cognitive outcomes in their offspring using the Bayley scales of infant development, third edition. In this extended follow-up of the same cohort of mother-child dyad, we propose to use ERP to study the long-term impact of maternal B12 supplementation on brain function in children at 72 months of age. We intend to use P300 and mismatch negativity (MMN) as measures of neurophysiological outcomes. The primary outcome of this study will be child neurophysiological measures (as measured by amplitude and latency of P300 and MMN) assessed at 72 months of age in children whose mothers received vitamin B12 compared with neurophysiological status of children whose mothers received placebo.

Ethics and dissemination The study was approved by the Institutional Ethical Board of St. John's Medical College and the Harvard School of Public Health Human Subjects Committee. Results obtained will be presented at national and international research meetings and published in peer-reviewed scientific journals.

Trial registration number NCT00641862.
Strengths and limitations of this study

- The present study examines the long-term effect of oral maternal B12 supplementation on brain functions in children.

- The capture of several known risk factors linked to neurodevelopmental outcomes in children is a particular strength of this study.

- The overall sample size of the study is modest.

- The present study examines the effect of a single micronutrient intervention on brain functions as opposed to multinutrient intervention.

\section{INTRODUCTION}

Vitamin B12 is a co-factor in the conversion of homocysteine to methionine. S-adenosylmethionine is a prominent methyl donor involved in the methylation of DNA and RNA. Impairment in this methylation could result in deficient production of myelin and thereby impact myelination in central nervous system (CNS). ${ }^{1}$ Animal studies using cycloleucine as an inhibitor of S-adenosylmethionine showed reduced methylation of the myelin basic protein providing a rational explanation for myelin lesions observed in cases of vitamin B12 deficiency. ${ }^{2}$ Myelination is involved in cognition, learning, skill development and memory. ${ }^{3}$ MRI-based studies have shown that myelination and white matter maturation correlate with development of specific cognitive functions. ${ }^{45}$

Vitamin B12-deficient diets or malabsorption are associated with delayed neurocognitive development ranging from irritability to failure to thrive, developmental regression and anorexia. ${ }^{6}$ Observational studies in children have shown that biochemical indices of poor vitamin B12 status are associated with 
poorer performance on tests of cognitive functions. ${ }^{78}$ A study in the Netherlands demonstrated an association between consumption of a macrobiotic diet (low in vitamin B12) during the first six years of life and poorer performance on measures of fluid intelligence, spatial ability and short-term memory during adolescence compared with children who were fed an omnivore diet. ${ }^{9}$ The beneficial effects of vitamin B12 status on cognitive performance in children were demonstrated in a randomised controlled trial in India ${ }^{10}$ and on motor development in two trials in Norwegian infants. ${ }^{11}{ }^{12}$ Several studies have noted that impaired maternal vitamin B12 status in pregnancy is associated with a greater risk of vitamin B12 deficiency in infants. ${ }^{13}{ }^{14}$ However, findings from studies that examined the association between maternal vitamin B12 status and cognitive function in children have been inconsistent. While some studies report an association between low maternal vitamin B12 status during pregnancy and suboptimal performance on cognitive tests in infants ${ }^{15}$ and young children, ${ }^{16}$ others did not. ${ }^{17}{ }^{18}$ Furthermore, it is difficult to reliably measure cognitive outcomes in infants and young children because changes in child development can be emergent, latent (not yet measurable), delayed or disordered and are rapidly changing. ${ }^{19}$

As vitamin B12 has an important role in myelination in the CNS, using neurophysiological-based assessments of brain function may yield additional information. Few studies have examined the effect of nutritional deficiencies on developing brain using event-related potentials (ERPs). ERPs reflect the electrical response of the cortex to cognitive, emotional or sensory events that are stimulus derived and synchronous or 'time locked' to particular stimuli or events. They are non-invasive, robust, directly reflect neural activity and have excellent temporal resolution, reflecting electrical activity in the order of milliseconds. ${ }^{20}$ Thus, ERPs provide a direct measure of the underlying neural activity.

Compared with healthy controls, infants with iron deficiency anaemia had delayed auditory brain responses ${ }^{2122}$ and delayed visual evoked potentials (EPs), ${ }^{23}$ suggestive of decreased myelination. In a study from Chile, delay in absolute latencies for auditory brain responses persisted at 4 years of age even after correction of iron deficiency anaemia during infancy, suggesting that iron deficiency can have long-lasting effects on the developing brain. ${ }^{24}$ Based on ERP studies, infants born to mothers with prenatal iron deficiency ${ }^{2526}$ and infants with iron deficiency anaemia had impaired recognition memory. ${ }^{27}$

In summary, the majority of studies using ERP have been performed in infants with iron deficiency anaemia. We are not aware of any studies that have examined the impact of maternal B12 supplementation on brain functions in children using an additional metric of ERPs.

We performed a double-blind randomised controlled trial of oral vitamin B12 during pregnancy and early lactation in south Indian women with a high prevalence of vitamin $\mathrm{B} 12$ deficiency. ${ }^{28}$ We have since been following children from this cohort with periodic cognitive

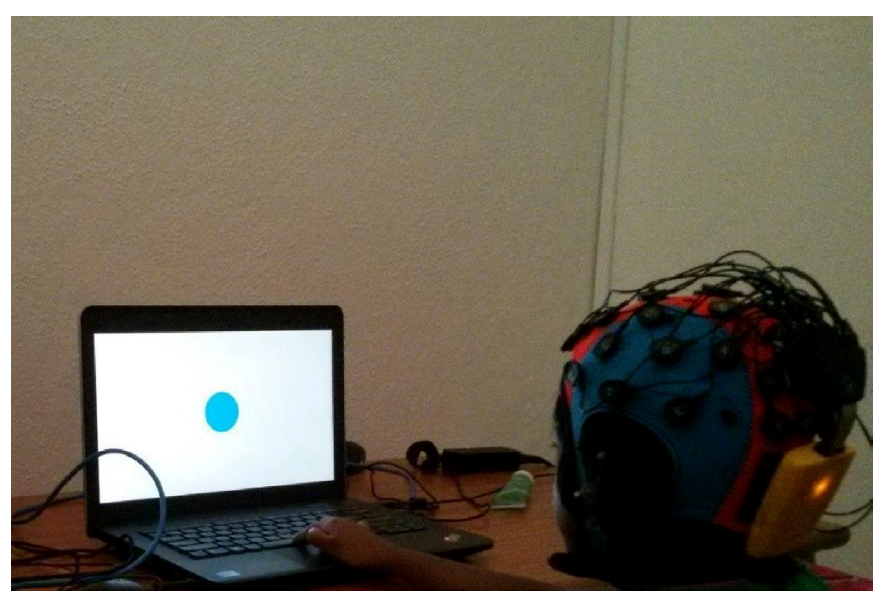

Figure 1 The Neuroelectrics ENOBIO system with the dry electrodes, electrode cap and the Neuroelectrics Instrument Controller device.

assessments (9, 18 and 30 months) using Bayley scales of infant development III(BSID-III). ${ }^{29}$ As part of consortium of studies whose primary objectives are to examine the long-term effects of B12 supplementation on neurodevelopmental outcomes and growth in children, ${ }^{30}$ we propose to examine the long-term impact of maternal B12 supplementation on child neurodevelopment using both conventional neuropsychological-based tests of cognitive abilities and measuring ERPs as a function of underlying neuronal activity. In this report, we present the methods of ERP acquisition using a novel wireless system, ENOBIO (Neuroelectrics, Barcelona). The ENOBIO Neuroelectrics Instrument Controller (NIC) consists of software that records continuous EEG data from a wireless Bluetooth device connected to 32 dry electrodes using an electrode cap (figure 1).

\section{Objective of the study}

To examine the effects of oral vitamin B12 daily supplements $(50 \mu \mathrm{g})$ to pregnant Indian women from 14 weeks gestational age through 6 weeks post partum on child neurophysiological function at 72 months.

\section{METHODS AND ANALYSIS \\ Present study}

This study is a follow-up of a placebo-controlled clinical trial of oral B12 supplementation ( $50 \mu$ g daily) beginning at $<14$ weeks of gestation through a 6 -week post partum on pregnant women in Bangalore. Recruitment and follow-up of mothers $(n=366)$ and their children took place at Hosahalli Hospital, a government-administered maternal facility in Bangalore that caters to women from lower socioeconomic backgrounds. At the time of recruitment in the parent study, a significant proportion $(51.1 \%)$ of pregnant women had B12 deficiency. Following maternal B12 supplementation, serum B12 levels were significantly higher in the intervention group and their infants. B12 levels in the breast milk were also significantly higher at 6 weeks $(\mathrm{p}<0.0005)$ in this group. ${ }^{28}$ 
We have been following up these mother-child dyads for 6 years with periodic neurocognitive assessments of children at 9 and 30 months. ${ }^{29} 31$

As part of the current study, the mother-child dyads are being assessed on tests from Kaufman Assessment Battery for Children, ${ }^{32}$ Koh's block design test ${ }^{33}$ and tests of verbal fluency, Vineland Social Maturity Scale ${ }^{34}$ and test of executive functions based on the BRIEF P. ${ }^{35}$ Socio-demographic details such as age, gestational age, household income, religion, parity, educational background of both parents and list of household assets will be estimated. Assessment of food security, home environment and parental behaviour would also be carried out.

Approximately $10 \mathrm{~mL}$ of blood will be obtained from the children by venipuncture and collected in both EDTA and plain vacutainers (BD Franklin Lakes, New Jersey, USA) that will be kept on ice until separation in a refrigerated centrifuge, usually within 4 hours. Haemoglobin and complete blood count will be analysed on whole-blood samples in an automated Coulter counter (ABX Pentra C+; Horiba Medicals, California, USA). The plasma and red blood cells will be separated and stored at $80^{\circ} \mathrm{C}$ until analysis for vitamin B12, total homocysteine (tHcy), methylmalonic acid (MMA) and erythrocyte folate concentrations. The plasma vitamin $\mathrm{B} 12$ will be measured by the electrochemiluminescence method (Roche Diagnostics Mannheim, USA). The measurement of tHcy and MMA will be performed by GC-MS (model 3800; Varian, Palo Alto, California, USA). Dietary intake in children will be measured by a 24-hour dietary recall form which has been previously validated among south Indian children. ${ }^{36}{ }^{37}$ Trained research personnel will record height, weight, body mass index, head circumference, waist circumference, hip circumference, chest circumference, mid-upper arm circumference, biceps skinfold, triceps skinfold, sub-scapular skinfold and the supra-iliac skinfold in participating children. ERP will be assessed at approximately 72 months of age to study the effects of maternal vitamin B12 supplementation on offspring brain function. For the current study, data collection started in January 2016 and we plan to complete data collection by December 2018.

\section{The experimental setting}

The neurophysiological assessments will be carried out in a central facility at St John's Research Institute. The background noise in the sound-proof room will be maintained below $40 \mathrm{~dB}$ throughout the experimental sessions. The tasks will be performed on a Lenovo ThinkPad with a 64-bit operating system. The participants will be seated in a comfortable, height-adjustable chair such that the computer screen is at the subject's eye level and about $60 \mathrm{~cm}$ from the subject. The subject will be examined in the mid-morning (between breakfast and lunch).

The cap with the dry electrodes will be snugly placed on the subject's scalp. A conductive gel provided with the ENOBIO kit will be applied on the right ear lobe which will be used as the electrode reference. A Bluetooth device will then be connected to the electrode scalp and paired with the laptop running the NIC software. The appropriate electrodes will be checked for maximum connectivity with the scalp to ensure proper transmission of the EEG signal to the electrodes. In addition to the subject, three persons comprising two research assistants and a parent or guardian of the participant will be present during the experimental session.

\section{Rationale for use of ENOBIO}

We chose ENOBIO over traditional EEG recording methods as it is a wireless system that does not require manual placement of wet electrodes and, thus, is better tolerated by the subject.

In addition, compared with traditional methods used for acquiring EEG signals, the ENOBIO system requires less preparatory time for ERP assessments. In traditional wired systems, the subject needs to be stationary to avoid disruption of recording. Collado-Mateo $e t a l^{38}$ have used ENOBIO system for non-stationary subjects performing tasks to understand brain activity during two physical balancing tasks. A study that explored the feasibility and test-retest reliability of EEG power using the wireless ENOBIO system with dry electrodes concluded that the test and retest reliability was 'excellent' with the mean Intraclass Correlation Coefficient $( \pm \mathrm{SD})$ of $0.95( \pm 0.02)$ for one of the balance tasks and $0.94( \pm 0.05)$ for the other balance task and the SEM \% oscillated from 0.57 to $1.02 .{ }^{38}$ Over the last decade, studies have used the ENOBIO system to measure ERP in children. Using this system, investigators studied changes in alpha, beta and theta EEG waves to understand attentional abilities in children aged 8-12 years with cerebral palsy (CP) compared with healthy controls. ${ }^{39}$ A recent multisite study of neurodevelopment in pre-school-age children, the INTERGROWTH 21 st Project, is also proposing to use the wireless electrode system to capture ERPs. ${ }^{40}$

\section{Experiments}

We propose to use ERPs to assess higher cognitive functions such as sensory discrimination of physical attributes of stimuli, attention and working memory in children aged 72 months born to mothers who received either oral vitamin B12 or placebo during pregnancy. ERPs generated $100 \mathrm{~ms}$ post-stimulus presentations are 'cognitive' and examine information processing because they reflect the manner in which a subject evaluates a stimulus. This is in contrast to early waves (components peaking within a $100 \mathrm{~ms}$ post-stimulus presentation) which are termed as sensory since they largely depend on the physical parameters of the stimulus. ${ }^{41}$

\section{Event-related potential tasks}

P300 and mismatch negativity (MMN) will be used, which are well-characterised 'cognitive' potentials and are known to reflect higher cognitive functions of attention and memory that are endogenous in nature. ${ }^{2042}$ The components of the ERPs that will be measured include 


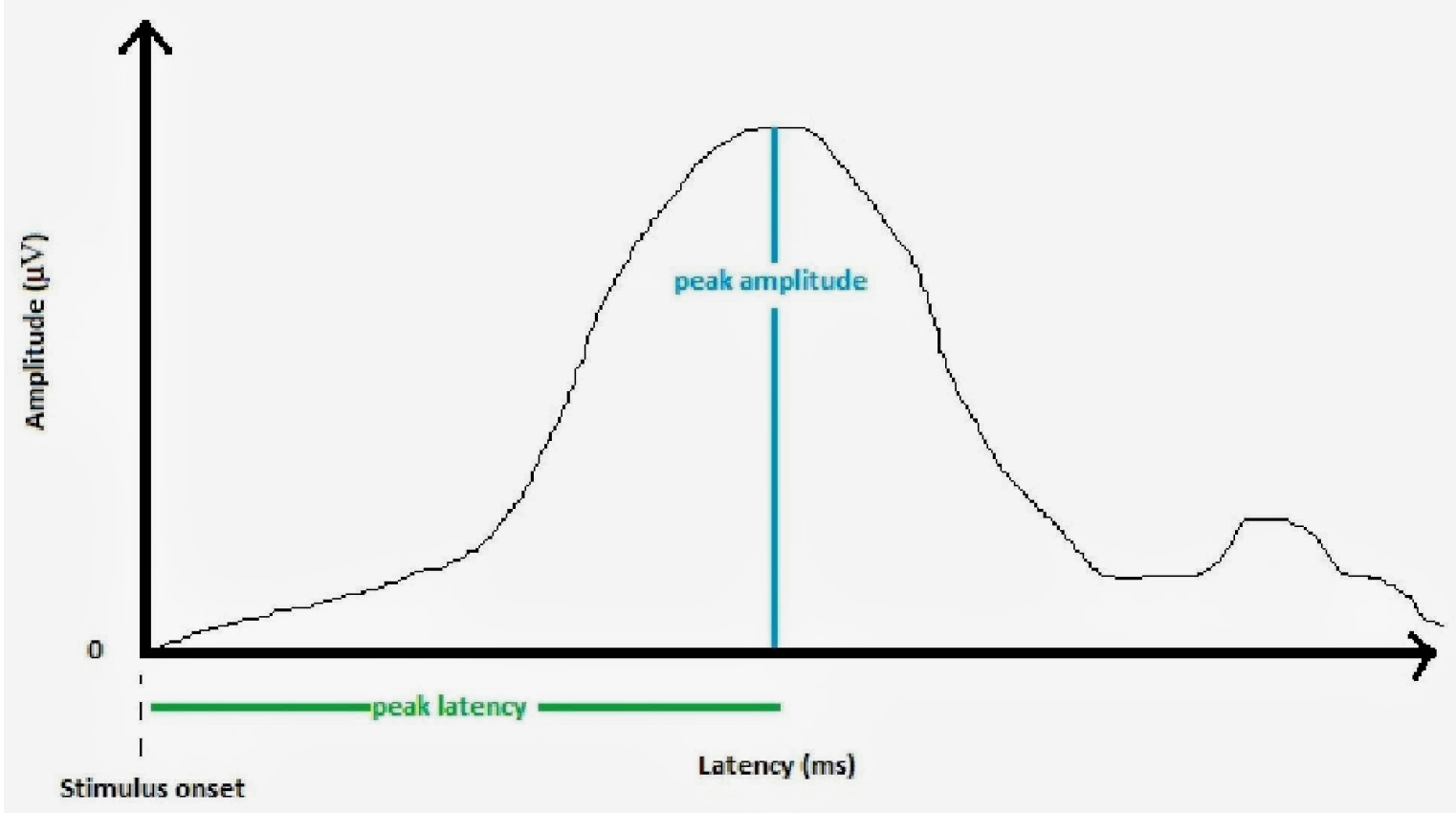

Figure 2 Event-related potentials: amplitude and latency.

the amplitude $(\mu \mathrm{V})$ and the latency (ms) (figure 2). Figure 3 illustrates the sequential process of electrophysiological experiment.

\section{The P300 experiment}

P300 is a 'positive' wave occurring at approximately $300 \mathrm{~ms}$ post-stimulus presentation. ${ }^{20}$ In tasks where the standard/target discrimination is more challenging, P300 is shown to be prominent over fronto/central region of the brain. ${ }^{43}$ P300 amplitude is influenced by attentional resource allocation (cognitive demands) while processing a task. ${ }^{44}$ Alterations in P300 are associated with impaired attention. ${ }^{42}$ Figure 4 shows the visual P300 stimuli and the task to be performed by the subject.

\section{P300 stimuli}

In the present study, three visual target stimuli will be presented on a white background. A blue circle $3.5 \mathrm{~cm}$ in diameter (the standard stimulus), same blue circle but $5 \mathrm{~cm}$ in diameter (the target stimulus) and a checkerboard image (the distractor stimulus) will be chosen as the target stimuli. Each stimulus will be presented on the video screen of the computer for $100 \mathrm{~ms}$ with an inter-stimulus interval (ISI) of $1000 \mathrm{~ms}$. The probabilities of the 'standard to target to distractor' ratio will be set at 80:10:10. Stimuli presentations will be randomised. A total of 1000 stimuli will be presented per session which is divided into five blocks of approximately $3.5 \mathrm{~min}$ each, with the above probabilities to minimise fatigue in the subjects. We chose the visual modality to test P300. ${ }^{45}$ in three stimulus oddball paradigm, using target, standard and infrequent non-target stimuli of 2000, 1000 and $500 \mathrm{~Hz}$ auditory task and ' $\mathrm{X}$ ', ' $\mathrm{O}$ ' and ' $\mathrm{H}$ ' respectively for the visual task showed that both auditory and visual stimulus modalities elicited an identical topography across both modalities of P300.

\section{The P300 task}

The task will be described to the participant. The subject will be instructed to keep their eyes fixed on the stimuli and respond with a button press only to the target stimulus and to ignore the standard and the distractor stimuli. A practice test will be carried out to ensure that the participant comprehends all the instructions. The subject will be instructed to use the index finger of their dominant hand to respond to the appropriate stimuli. The session is expected to last about 20 min for all five blocks.

\section{The MMN experiment}

MMN is a neural response seen as a result of discrimination of a particular aspect of an auditory stimulus from the preceding one. It peaks $100-300 \mathrm{~ms}$ after the onset of stimulus. ${ }^{46}$ It represents a 'pre-attentive' memory-based comparison process between the neural representation of incoming deviant auditory stimulus and that of previous regular stimuli. ${ }^{46}$ The primary auditory cortex, the dorsolateral prefrontal cortex and the frontal region of the brain are the most likely cerebral sources of MMN. ${ }^{48-50}$ MMN reflects cognitive state of the early stage of obligatory auditory attention in infants and children. MMN is robustly elicited not only when a subject is inattentive to the auditory stimuli but maybe engaged in a cognitively demanding task in another modality. ${ }^{51}$ MMN has been studied in various neuropsychiatric conditions such as schizophrenia and dyslexia, and in subjects with auditory processing disabilities. ${ }^{47}$ Figure 5 shows the auditory stimuli and the task to be performed by the subject. 


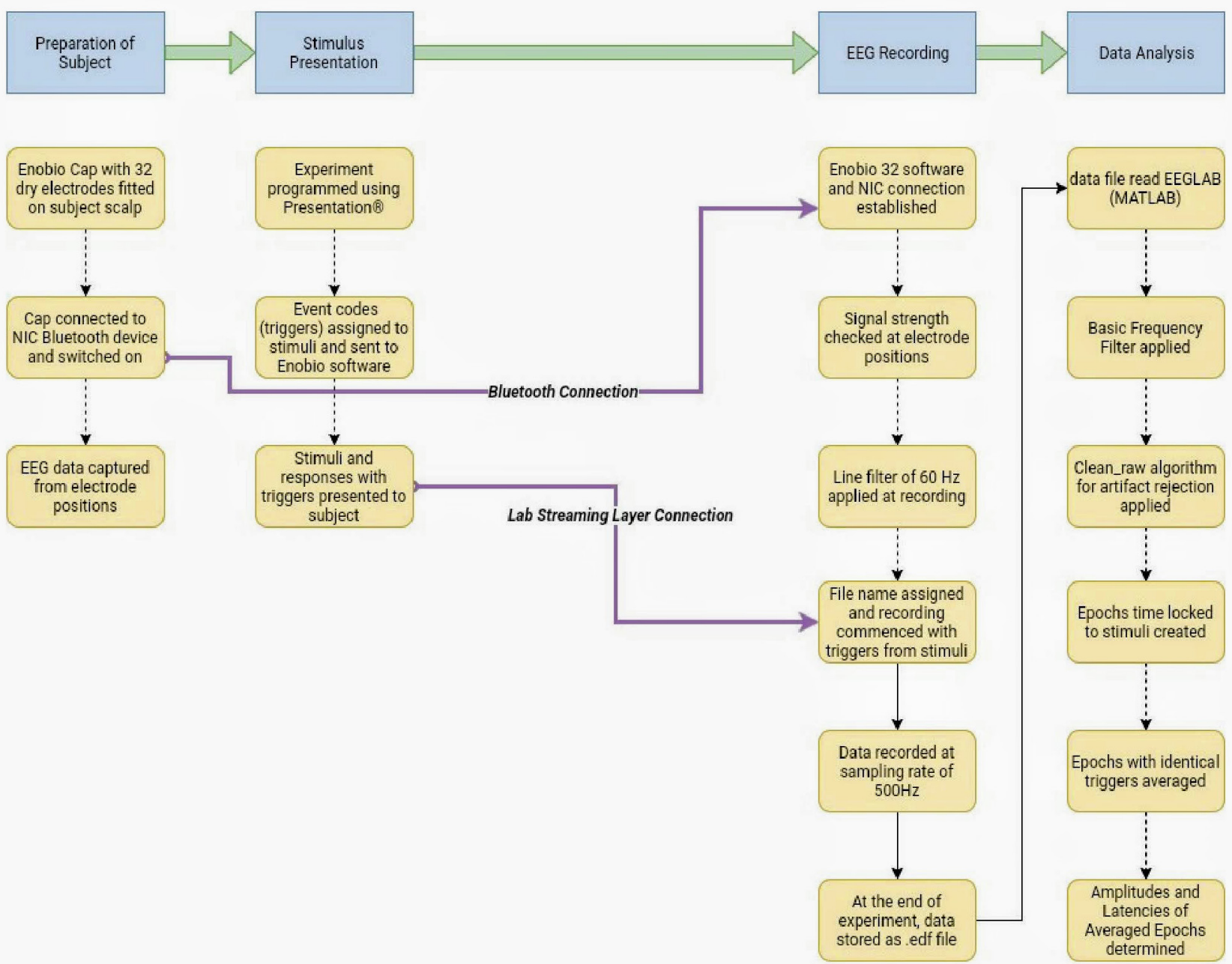

Figure 3 Workflow of electrophysiological experiments along with information about system connections.

\section{The MMN stimuli}

The $1000 \mathrm{~Hz}$ tone (the standard), $2000 \mathrm{~Hz}$ tone (deviantMMN 1), $1100 \mathrm{~Hz}$ tone (deviant-MMN 2) and $1010 \mathrm{~Hz}$ tone (deviant-MMN 3) will be chosen as the auditory stimuli for the three MMN tasks. The duration of each stimulus will be $100 \mathrm{~ms}$ long, with $5 \mathrm{~ms}$ each of rise and fall of the auditory stimuli. The ISI of $700 \mathrm{~ms}$ will be maintained for all the three MMN experiments. The auditory stimuli will have a decibel range of 75-80 dB. The 'standard to deviant' probability is set at 80:20 and a total of 1000 stimuli will be presented per MMN task each of which will last up to $10 \mathrm{~min}$, totalling $30 \mathrm{~min}$ for all three MMN tasks.

\section{The MMN task}

The subject will be seated with a laptop playing a mute engaging cartoon video to divert attention from the experimental task. The MMN experiment consists of presentation of standard stimuli at $1000 \mathrm{~Hz}$ compared with deviant stimuli of three different frequencies (1010, 1100 and $2000 \mathrm{~Hz}$ ). The $1000 \mathrm{~Hz}$ tone will be maintained as a standard for all the three MMN experiments and only the frequency of the deviant tone will differ across the MMN experiments.
The stimuli will be presented to the subject with over-ear 'Kidz Gear Headphones' that are specially designed for smaller head sizes. The headphones are padded for comfort and to maintain appropriate acoustics. The order of MMN experiments presented will be counterbalanced across all subjects to ensure that possible order effects are evenly distributed throughout the experiment. ${ }^{52}$ Subjects will be instructed to pay no attention to the auditory stimuli and encouraged to watch the cartoon video while the experiments are in progress.

\section{ERP set-up}

\section{Stimulus presentation software}

The experiment will be performed using Presentation software (V.18.0, Neurobehavioral Systems, Berkeley, California, USA) which is a programmable stimulus delivery and experimental control program that delivers visual and auditory stimuli with great accuracy and identifies event logging. Experiments will be programmed using the Presentation Control Language and the Presentation Control Application Programming Interface that enables loading and running of the experiments. The program is also capable of interfacing with other devices to send and receive data. 


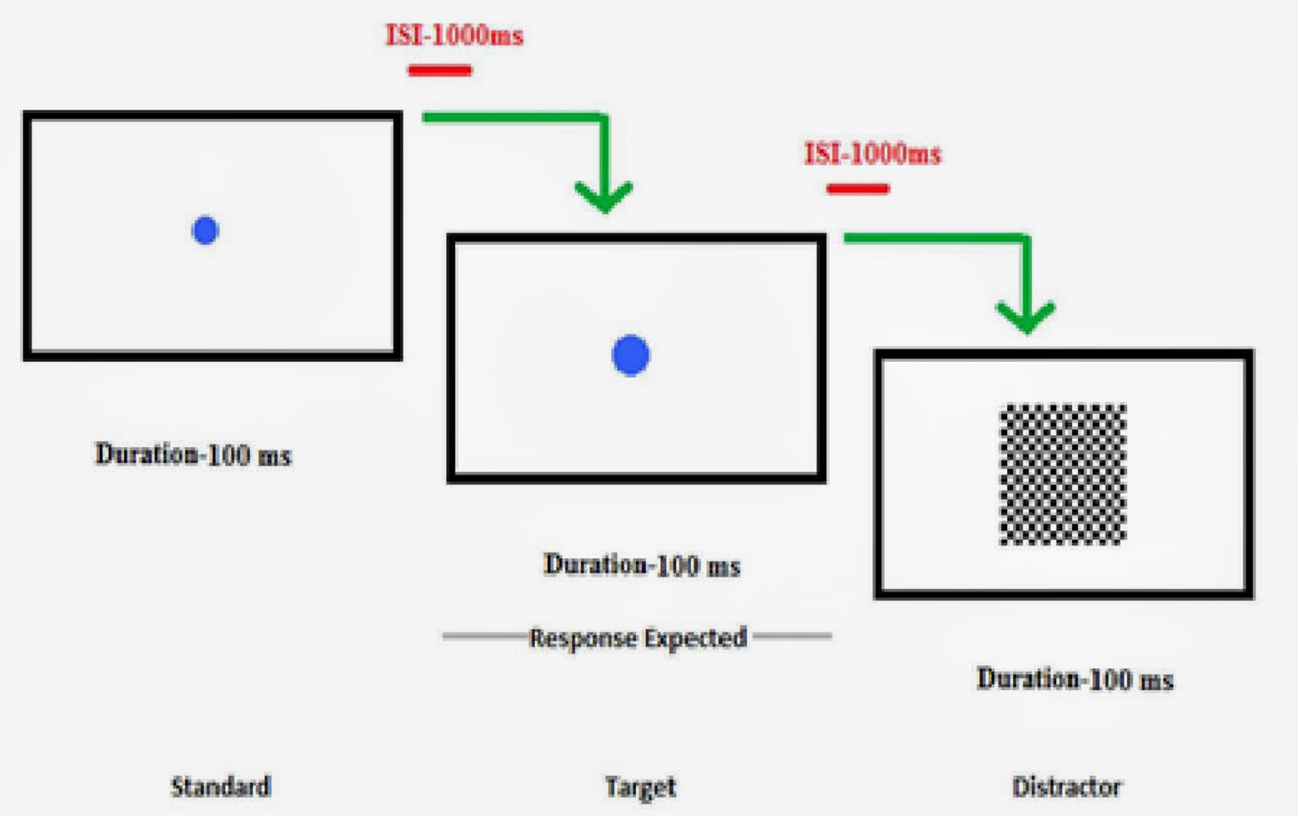

Figure 4 P300 stimuli and task. This figure shows the stimuli, the duration of the presentation of each stimuli and the interstimulus interval (ISI) of the P300 experiment. The three stimuli are presented at complete random in the ratio of 80:10:10 (standard:target:distraction).

\section{EEG data recording}

The EEG data will be recorded using ENOBIO which is a wearable, wireless electrophysiology sensor system. It is integrated with a powerful user interface to record and visualise 24-bit EEG data at 500 samples per second using the NIC software. In addition, the ENOBIO system is capable of integrating with the Presentation system using Lab Streaming Layer to record a stimulus from the stimulus delivery system. Thirty-two dry electrodes will be placed as per the International 10/20 system, a commonly used EEG electrode placement system for correlating external skull locations to underlying cortical areas using an electrode cap. ${ }^{53}$ The NIC Bluetooth Device will be used to record EEG signals from the 32 electrodes. Software-enabled line noise filter of $60 \mathrm{~Hz}$ will be applied to eliminate external electrical interference during the recording of the EEG data. The EEG recording will begin just prior to stimulus presentation and will be stopped just after the end of the experiment to prevent any loss of stimulus locked data. The data will be saved and stored on a drive as a European Data Format file (EDF). The EDF is a widely accepted standard for the exchange of EEG data between different equipment and laboratories. ${ }^{54}$

\section{EEG data analysis}

The analysis of the raw EEG data stored by the NIC system will be done using EEGLAB and ERPLAB for artefact rejection and data averaging and then for estimating the resultant peak amplitude and latencies of the ERPs. ERPLAB, which is closely integrated with EEGLAB, is a freely available open source toolbox that is used to process and analyse ERPs in the MATLAB environment (MATLAB r2015a, license number 1111289). ERPLAB adds on to EEGLAB's EEG processing functions that enables artefact detection, sorting of events, re-referencing as well as averaging EEG segments to create averaged ERPs, and different waves and plotting of these waveforms among other functions. In addition, the MATLAB environment makes it convenient to create small programs or scripts that enable an automated data processing with the flexibility of changing the script for data analysis of multiple subjects. ${ }^{55}$

\section{ERP data analysis}

\section{Analysis of P300 data}

The P300 data from the five blocks will be merged to create a single continuous EDF file. The data will be initially subjected to a Butterworth type of bandpass frequency filter of $0.1 \mathrm{~Hz}$ (highpass) $-30 \mathrm{~Hz}$ (lowpass) for artefact rejection. ${ }^{56}$ The P300 data will be analysed from the frontal midline electrode ' $\mathrm{Fz}$ ' as the P300 has been shown to be prominent in the fronto/central region. An algorithm called 'clean_raw data' using a $0.25-0.75 \mathrm{~Hz}$ transition band that cleans continuous EEG data using artefact subspace reconstruction method will be used for artefact rejection. After the removal of artefacts, epochs (time segments of waveforms) of $-100 \mathrm{~ms}$ (100 ms prior to the stimulus presentation) to $900 \mathrm{~ms}(900 \mathrm{~ms}$ post-stimulus presentation) will be created for generating P300 data. The epochs from the standard and deviant stimuli will be averaged to create the standard and deviant EPs. The amplitude and latencies of P300 will be calculated at the previously chosen $\mathrm{Fz}$ electrode position. Peak amplitudes and latencies of P300 will be estimated using the ERP measurement tool with predetermined time windows. For P300 data, -100 to $600 \mathrm{~ms}$ time windows will be chosen, and with the help of this function, the 


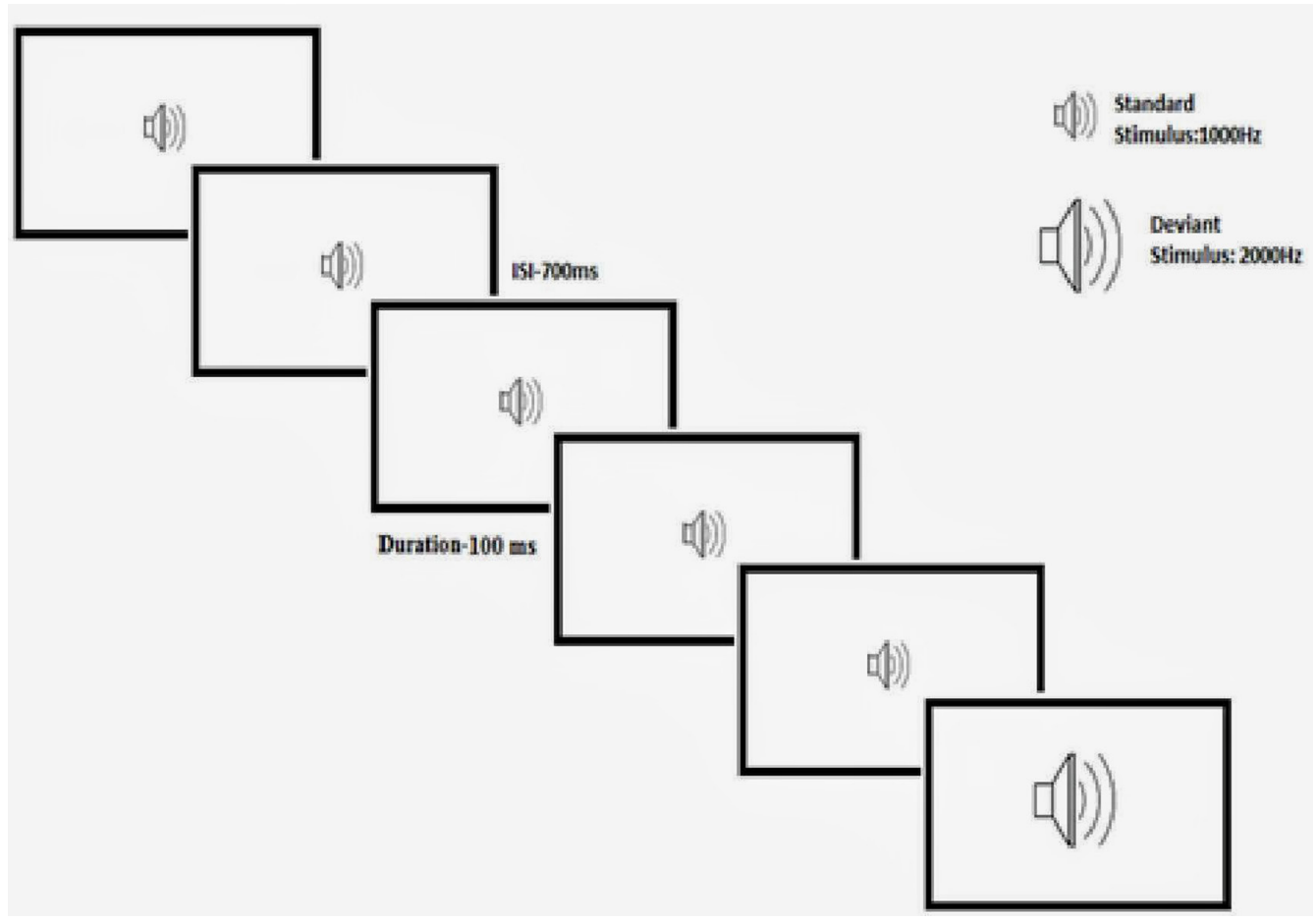

Figure 5 Mismatch negativity (MMN) stimuli and task. This figure shows the stimuli, the duration of the presentation of each stimuli and the inter-stimulus interval of the MMN experiment. The two stimuli are presented at complete random in the ratio of 80:20 (standard:deviant).

amplitude $(\mu \mathrm{V})$ and latency $(\mathrm{ms})$ of the highest positive peak will be determined.

\section{Analysis of MMN data}

The EDF file of the MMN experiment will be initially loaded onto the EEGLAB interface. Similar to the P300 analysis, the EEG data will be initially subjected to Butterworth filter of $0.1 \mathrm{~Hz}$ (highpass) $-30 \mathrm{~Hz}$ (lowpass). As with the P300 analysis, the Fz electrode will be chosen for the analysis of the MMN data as the frontal region is the most likely the cerebral source of MMN ${ }^{44-46}$ After the application of the bandpass filter, the 'clean_rawdata' method will be applied for artefact rejection. After the removal of artefacts, epochs of $-100 \mathrm{~ms}$ ( $100 \mathrm{~ms}$ prior to the stimulus presentation) to $600 \mathrm{~ms}$ (600 ms post-stimulus presentation) will be created for generating MMN data. EPs are created by averaging the epochs determined from standard and the deviant stimuli independently. To characterise the MMN, difference waves (where the standard stimulus ERP will be subtracted from the deviant stimuli ERP) will be created and peak amplitude and latencies will be determined. The amplitude and latencies will be calculated for the $\mathrm{Fz}$ channel. Peak amplitudes and latencies will be determined using the ERP Measurement Tool with predetermined time windows. A time window of -100 to $350 \mathrm{~ms}$ for the MMN data will be chosen, and the amplitudes and latencies for the most negative peak in the time window will be determined.

\section{Statistical considerations}

\section{Determination of primary outcomes}

The primary endpoint will be ERP measures at 72 months which would be amplitude and latency of P300 and MMN. We hypothesise that children born to mothers who received $50 \mu \mathrm{g}$ of oral vitamin $\mathrm{B} 12$ will have a higher amplitude and shorter latency of P300 and MMN compared with children of mothers who received placebo.

\section{Sample size justification}

We plan to evaluate all available children born to the 366 women in the B12 supplementation trial for neurodevelopmental outcomes through $61 / 2$ years of age. A sample size of 60 per arm will provide $90 \%$ power to detect a standardised effect size of 0.6 or higher in latency with a two-sided two-sample t test at 0.05 significance level.

\section{General analytic strategy}

Intent-to-treat analysis of treatment effects will be the primary analysis strategy for all endpoints. All tests will be two-sided at 0.05 significance level. The primary comparison will use a two-sample $t$ test. Wilcoxon rank-sum test will be used if the scores substantially deviate from the normal distribution. The association between markers of child vitamin B12 status (at 72 months) and ERP measures (at 72 months) will be evaluated by Pearson's correlation coefficients (if the outcomes are normally distributed) or Spearman's rank correlation coefficients (if the outcomes are not normally distributed). Multiple linear regression with ERP as the dependent variable will be performed to further assess these associations in a multivariable model. 
The association between measured outcome of interest and baseline risk factors, as well as longitudinally measured risk factors including dietary intake, nutritional status and home environmental factors, will be examined using generalised linear models.

We will include interaction terms in multiple models to examine whether B12 supplementation is more effective in certain subpopulations. Due to limited statistical power, all potential effect modifiers will be dichotomised into two groups with relatively balanced sample sizes. The appropriate cut point for continuous or ordinal covariates may be the median value, 1st or 3rd quartile, or commonly used cut-offs.

The primary intent of this study is to measure the effect of maternal vitamin B12 supplementation on the brain function of the offspring using neurophysiological measures. Electrophysiological methods like ERPs or EPs have proved to be reliable in measuring neural correlates of cognitive function. The extent of research using ERPs to study the impact of vitamin B12 on brain functions in children remains limited. We will attempt to bridge this knowledge gap by using the ERPs P300 and MMN as measures of sensory discrimination, attention and working memory, in addition to neuropsychological assessments.

\section{Patient and public involvement}

The present study is a follow-up of a double-blind placebo controlled trial of B12 supplementation during pregnancy. Our team has been following up these motherchild dyads for 6 years and our research team has been in constant contact with participants for cognitive assessments. The participants have been receptive to the feedback given during the original trial and have expressed their interest in examining the long-term effects of supplementation. We will discuss strategies to provide information to the participants at the completion of the present study.

\section{Ethics and dissemination}

The study was approved by the Institutional Ethical Board of St. John's Medical College and the Harvard School of Public Health Human Subjects Committee. Written informed consent will be taken from the parents of the participating children. Verbal assent will be taken from the participating children. Results obtained will be presented at national and international research and policy meetings, and published in peer-reviewed scientific journals.

The present study is an extended follow-up of children examining the impact of maternal vitamin B12 supplementation on neurodevelopmental and neurophysiological outcomes in children. The extended nature of follow-ups is a particular strength of the study along with the capture of several known risk factors linked to neurodevelopmental outcomes in children. However, loss to follow-up and attrition can influence the proposed outcomes but we do not expect this to differ between the two arms of the study.

The results from the study could further our understanding of the role of vitamin B12 in brain functions in children and be helpful in formulating policy decisions on maternal nutrient supplementation and its impact on neurodevelopmental outcomes in children.

\section{Author affiliations}

${ }^{1}$ Division of Mental Health and Neurosciences, St. John's Research Institute, Bangalore, Karnataka, India

${ }^{2}$ Department of Biostatistics, St John's Medical College, Bangalore, Karnataka, India ${ }^{3}$ Centre for International Health, University of Bergen, Bergen, Norway

${ }^{4}$ Division of Nutrition, St. John's Research Institute, Bangalore, Karnataka, India

${ }^{5}$ Center for Nutrition, Division of Gastroenterology, Hepatology and Nutrition, Boston Children's Hospital, Boston, Massachusetts, USA

${ }^{6}$ St John's Research Institute, St John's National Academy of Health Sciences, Bangalore, Karnataka, India

Acknowledgements The authors thank all the participants who have agreed to be a part of our study and graciously gave their consent for the tests. They are grateful to Dr B Nirmala for helping obtain all the clearances to conduct the study at Hosahalli Hospital. They thank Ms P. Vijaya, Ms S. Surekha and Ms C. Darshini for technical support. The authors thank Ms D. Sarita for conducting the analyses of total homocysteine and methylmalonic acid, and Ms R. Shanti and Ms B. Beena for conducting the analyses of vitamin B12.

Contributors KS, AVK, CD and TAS conceptualised the project. MJ designed the ERP experimental sessions. SA conducted the electrophysiological experiment and collected data. KS and ST are responsible for setting up the study. TT designed the statistical analyses. SA, ST and KS wrote the paper. KS had the primary responsibility for the final content. All authors have read and approved the final manuscript.

Funding The present study is supported by the Norwegian Research Council (grant number 234495). The parent trial was supported by the Indian Council of Medical Research grant 5/7/192/06-RHN and the US National Institutes of Health grant R03 HD054123. CD is supported in part by NIH grants K24DK104676 and 2P30 DK040561.

Competing interests None declared.

Patient consent for publication Not required.

Provenance and peer review Not commissioned; externally peer reviewed.

Open access This is an open access article distributed in accordance with the Creative Commons Attribution Non Commercial (CC BY-NC 4.0) license, which permits others to distribute, remix, adapt, build upon this work non-commercially, and license their derivative works on different terms, provided the original work is properly cited, appropriate credit is given, any changes made indicated, and the use is non-commercial. See: http://creativecommons.org/licenses/by-nc/4.0/.

\section{REFERENCES}

1. Allen RH, Stabler SP, Savage DG, et al. Metabolic abnormalities in cobalamin (vitamin B12) and folate deficiency. Faseb $J$ 1993;7:1344-53.

2. Small DH, Carnegie PR, Anderson RM. Cycloleucine-induced vacuolation of myelin is associated with inhibition of protein methylation. Neurosci Lett 1981;21:287-92.

3. Fields RD. White matter in learning, cognition and psychiatric disorders. Trends Neurosci 2008;31:361-70.

4. Mabbott DJ, Noseworthy M, Bouffet E, et al. White matter growth as a mechanism of cognitive development in children. Neuroimage 2006;33:936-46.

5. Nagy Z, Westerberg $\mathrm{H}$, Klingberg T. Maturation of white matter is associated with the development of cognitive functions during childhood. J Cogn Neurosci 2004;16:1227-33.

6. Dror DK, Allen LH. Effect of vitamin B12 deficiency on neurodevelopment in infants: current knowledge and possible mechanisms. Nutr Rev 2008;66:250-5.

7. Strand TA, Taneja S, Ueland PM, et al. Cobalamin and folate status predicts mental development scores in North Indian children 12-18 mo of age. Am J Clin Nutr 2013;97:310-7. 
8. Kvestad I, Hysing M, Shrestha M, et al. Vitamin B-12 status in infancy is positively associated with development and cognitive functioning 5 y later in Nepalese children. Am J Clin Nutr 2017;105:1122-31.

9. Louwman MW, van Dusseldorp M, van de Vijver FJ, et al. Signs of impaired cognitive function in adolescents with marginal cobalamin status. Am J Clin Nutr 2000;72:762-9.

10. Kvestad I, Taneja S, Kumar T, et al. Vitamin b12 and folic acid improve gross motor and problem-solving skills in young north indian children: a randomized placebo-controlled trial. PLOS One 2015;10:e0129915.

11. Torsvik I, Ueland PM, Markestad T, et al. Cobalamin supplementation improves motor development and regurgitations in infants: results from a randomized intervention study. Am J Clin Nutr 2013;98:1233-40.

12. Torsvik IK, Ueland PM, Markestad T, et al. Motor development related to duration of exclusive breastfeeding, $\mathrm{B}$ vitamin status and B12 supplementation in infants with a birth weight between $2000-$ $3000 \mathrm{~g}$, results from a randomized intervention trial. BMC Pediatr 2015; $15: 218$.

13. Muthayya S, Dwarkanath P, Mhaskar M, et al. The relationship of neonatal serum vitamin B12 status with birth weight. Asia Pac J Clin Nutr 2006;15:538-43.

14. Finkelstein JL, Kurpad AV, Thomas $T$, et al. Vitamin $B_{12}$ status in pregnant women and their infants in South India. Eur J Clin Nutr 2017;71:1046-53.

15. del Río Garcia C, Torres-Sánchez L, Chen J, et al. Maternal MTHFR $677 \mathrm{C}>\mathrm{T}$ genotype and dietary intake of folate and vitamin $\mathrm{B}(12)$ : their impact on child neurodevelopment. Nutr Neurosci 2009;12:13-20.

16. Bhate V, Deshpande S, Bhat D, et al. Vitamin B12 status of pregnant Indian women and cognitive function in their 9-year-old children. Food Nutr Bull 2008;29:249-54

17. Veena SR, Krishnaveni GV, Srinivasan K, et al. Higher maternal plasma folate but not vitamin B-12 concentrations during pregnancy are associated with better cognitive function scores in 9- to 10- yearold children in South India. J Nutr 2010;140:1014-22.

18. Bonilla C, Lawlor DA, Taylor AE, et al. Vitamin B-12 status during pregnancy and child's IQ at age 8: a Mendelian randomization study in the Avon longitudinal study of parents and children. PLoS One 2012; 7:e51084.

19. Marks K, Glascoe FP, Aylward GP, et al. The thorny nature of predictive validity studies on screening tests for developmentalbehavioral problems. Pediatrics 2008;122:866-8.

20. Hajcak G, MacNamara A, Olvet DM. Event-related potentials, emotion, and emotion regulation: an integrative review. Dev Neuropsychol 2010;35:129-55.

21. Roncagliolo M, Garrido M, Walter T, et al. Evidence of altered central nervous system development in infants with iron deficiency anemia at $6 \mathrm{mo}$ : delayed maturation of auditory brainstem responses. Am J Clin Nutr 1998;68:683-90.

22. Kürekçi $A E$, Sarici $S U$, Karaoglu A, et al. Effects of iron deficiency versus iron deficiency anemia on brainstem auditory evoked potentials in infancy. Turk J Pediatr 2006;48:334.

23. Monga $M$, Walia $V$, Gandhi $A$, et al. Effect of iron deficiency anemia on visual evoked potential of growing children. Brain Dev 2010;32:213-6.

24. Algarín C, Peirano P, Garrido M, et al. Iron deficiency anemia in infancy: long-lasting effects on auditory and visual system functioning. Pediatr Res 2003;53:217-23.

25. Deregnier RA, Nelson CA, Thomas KM, et al. Neurophysiologic evaluation of auditory recognition memory in healthy newborn infants and infants of diabetic mothers. J Pediatr 2000;137:777-84.

26. Nelson CA, Wewerka SS, Borscheid AJ, et al. Electrophysiologic evidence of impaired cross-modal recognition memory in 8-monthold infants of diabetic mothers. J Pediatr 2003;142:575-82.

27. Burden MJ, Westerlund AJ, Armony-Sivan R, et al. An event-related potential study of attention and recognition memory in infants with iron-deficiency anemia. Pediatrics 2007;120:e336-e345.

28. Duggan C, Srinivasan K, Thomas T, et al. Vitamin B-12 supplementation during pregnancy and early lactation increases maternal, breast milk, and infant measures of vitamin B-12 status. $J$ Nutr 2014;144:758-64.

29. Srinivasan K, Thomas T, Kapanee AR, et al. Effects of maternal vitamin $B 12$ supplementation on early infant neurocognitive outcomes: a randomized controlled clinical trial. Matern Child Nutr 2017;13:e12325.

30. Winje BA, Kvestad I, Krishnamachari S, et al. Does early vitamin $B_{12}$ supplementation improve neurodevelopment and cognitive function in childhood and into school age: a study protocol for extended follow-ups from randomised controlled trials in India and Tanzania. BMJ Open 2018;8:e018962

31. Thomas $\mathrm{S}$, Thomas $\mathrm{T}$, Bosch RJ, et al. Effect of maternal vitamin b12 supplementation on cognitive outcomes in south indian children: a randomized controlled clinical trial. Matern Child Health $J$ 2018;12:1-9.

32. Kaufman AS, Kaufman NL. Kaufman assessment battery for children New York: John Wiley \& Sons, Inc, 1983.

33. Kohs SC. Intelligence measurement. New York: Macmillan Company, 1923.

34. Doll EA. Vineland social maturity scale: Condensed manual of directions. Minneapolis: Educational Test Bureau, American Guidance Service, 1965

35. Gioia GA, Andrwes K, Isquith PK. Behavior rating inventory of executive function-preschool version (BRIEF-P. Odessa, FL: Psychological Assessment Resources, 1996.

36. Bharathi AV, Kurpad AV, Thomas T, et al. Development of food frequency questionnaires and a nutrient database for the Prospective Urban and Rural Epidemiological (PURE) pilot study in South India: methodological issues. Asia Pac J Clin Nutr 2008;17:178-85.

37. Swaminathan S, Thomas T, Yusuf S, et al. Clustering of diet, physical activity and overweight in parents and offspring in South India. Eur $J$ Clin Nutr 2013;67:128-34.

38. Collado-Mateo D, Adsuar JC, Olivares PR, et al. Using a dry electrode EEG device during balance tasks in healthy youngadult males: Test-retest reliability analysis. Somatosens Mot Res 2015;32:219-26.

39. Perales FJ, Amengual E. Combining EEG and Serious Games for Attention Assessment of Children with Cerebral Palsy. In: Pons JL, Torricelli D, Pajero M, eds. Converging Clinical and Engineering Research on Neurorehabilitation II. Cham: Springer, 2017:395-9.

40. Fernandes M, Stein A, Newton CR, et al. The INTERGROWTH-21st Project Neurodevelopment Package: a novel method for the multidimensional assessment of neurodevelopment in pre-school age children. PLoS One 2014;9:e113360.

41. Sur S, Sinha VK. Event-related potential: An overview. Ind Psychiatry J 2009;18:70.

42. Duncan CC, Barry RJ, Connolly JF, et al. Event-related potentials in clinical research: guidelines for eliciting, recording, and quantifying mismatch negativity, P300, and N400. Clin Neurophysiol 2009;120:1883-908.

43. Comerchero MD, Polich J. P3a and P3b from typical auditory and visual stimuli. Clin Neurophysiol 1999;110:24-30.

44. Polich J. Updating P300: an integrative theory of P3a and P3b. Clin Neurophysiol 2007;118:2128-48.

45. Katayama J, Polich J. Auditory and visual P300 topography from a 3 stimulus paradigm. Clin Neurophysiol 1999;110:463-8.

46. Pakarinen S, Takegata R, Rinne T, et al. Measurement of extensive auditory discrimination profiles using the mismatch negativity (MMN) of the auditory event-related potential (ERP). Clin Neurophysiol 2007;118:177-85.

47. Shelley AM, Ward PB, Catts SV, et al. Mismatch negativity: an index of a preattentive processing deficit in schizophrenia. Biol Psychiatry 1991;30:1059-62.

48. Cowan N, Winkler I, Teder W, et al. Memory prerequisites of mismatch negativity in the auditory event-related potential (ERP). $J$ Exp Psychol 1993;19:909-21.

49. Woods DL, Alho K, Algazi A. Stages of auditory feature conjunction: an event-related brain potential study. J Exp Psychol Hum Percept Perform 1994;20:81-94.

50. Alho K. Cerebral generators of mismatch negativity (MMN) and its magnetic counterpart (MMNm) elicited by sound changes. Ear Hear 1995; 16:38-51.

51. Nelson CA, McCleery JP. Use of event-related potentials in the study of typical and atypical development. J Am Acad Child Adolesc Psychiatry 2008;47:1252-61.

52. Mackay WE, Appert C, Beaudouin-Lafon M, et al. Touchstone: exploratory design of experiments. ACM 2007:1425-34.

53. Herwig U, Satrapi $P$, Schönfeldt-Lecuona $C$. Using the international 10-20 EEG system for positioning of transcranial magnetic stimulation. Brain Topogr 2003;16:95-9.

54. Kemp B, Olivan J. European data format 'plus' (EDF+), an EDF alike standard format for the exchange of physiological data. Clin Neurophysiol 2003;114:1755-61.

55. Lopez-Calderon J, Luck SJ. ERPLAB: an open-source toolbox for the analysis of event-related potentials. Front Hum Neurosci 2014;8:213.

56. Butterworth S. On the theory of filter amplifiers. Wireless Engineer 1930;7:536-41. 